\title{
LOCALIZAÇÃO DE FALTAS EM LINHAS DE TRANSMISSÃO COM MÚLTIPLAS DERIVAÇÕES UTILIZANDO DADOS DE SOMENTE DOIS TERMINAIS
}

\author{
Silvio Giuseppe Di Santo*
}

\author{
Carlos Eduardo de Morais Pereira*
}

Luiz Cera Zanetta Jr*

*PEA-POLI-USP

Caixa Postal 61548

CEP: 05424-970 - São Paulo SP

\section{RESUMO}

Um novo método de localização de faltas em linhas de transmissão continuamente transpostas e com múltiplas derivações é apresentado neste artigo. O mesmo é baseado nos fasores de tensão e corrente, pré-falta e pós-falta, medidos em regime permanente nos terminais local e remoto, para determinar as informações de falta, tais como: o trecho de ocorrência, a distância de falta, ou seja, o ponto de ocorrência, e a resistência.

A principal característica deste novo método é o processo de varredura da rede, onde a falta é localizada dentro de cada um dos trechos da linha, buscando-se minimizar por meio de uma função objetivo, as diferenças entre as tensões e correntes medidas e calculadas nos terminais local e remoto.

O erro calculado pela função objetivo, no caso de a falta estar em um determinado trecho, é comparado com o erro obtido para o trecho anterior, sendo o trecho com o maior erro descartado.

No final do processo de varredura, o método de localização de faltas tem como resultado o trecho de ocorrência, a distância e a resistência da falta.

Artigo submetido em 26/11/2009 (Id.: 01086)

Revisado em 01/02/2010, 27/05/2010

Aceito sob recomendação do Editor Associado Prof. Julio Cesar Stacchini Souza
Os resultados das simulações mostram a eficiência e a precisão do método proposto frente a variação da distância, resistência e tipo da falta.

PALAVRAS-CHAVE: Localização de faltas, Sistemas de Potência, Otimização.

\section{ABSTRACT}

Fault location on multiterminal transmission lines using only two terminals data

A new multiterminal continuously transposed transmission lines fault location method is presented in this paper, which it is based on steady-state pre-fault and post-fault voltage and current measured phasors at local and remote terminals to determine the fault information, which are: the section occurrence, the distance, or the occurrence point, and resistance.

The main characteristic of this new method is the process of networkscanning, where the fault is located within each line section, trying to minimize by means of an objective function, the differences between the voltages and currents measured and calculated in local and remote terminals.

The the error calculated by the goal function, in case of the fault to be in a certain section, is compared with the error obtained for the previous section, so the section with the 
largest error is dismissed.

At the end of the scanning process, the method of fault location presents the section of fault occurrence, distance and resistance.

The results obtained from the simulations show the efficiency and accuracy of the proposed method for the fault distance, resistance and type variation.

KEYWORDS: Fault location, Power systems, Optimization.

\section{INTRODUÇÃO}

A correta localização de faltas em linhas de transmissão é uma importante necessidade, uma vez que o tempo de reparo destas, no caso de faltas permanentes, afeta consideravelmente a confiabilidade do sistema bem como os prejuízos causados pela parada de processos industriais.

Os métodos de localização de faltas que utilizam fasores em regime permanente são divididos em métodos que utilizam dados de apenas um terminal e métodos que utilizam dados de vários terminais para a determinação do ponto da falta.

Alguns dos métodos de localização de faltas que utilizam dados de um terminal são os apresentados pelas referências (Takagi et al, 1981; Jeyasurya e Rahman, 1991; Johns et al., 1995; Novosel et al., 1996). Os métodos que utilizam dados de dois terminais, em linhas sem derivações, são os apresentados pelas referências (Gopalakrishnan et al., 2000; Brahma e Girgis, 2004; Faybisovich e Khoroshev, 2008; Fulczyk et al., 2008; Wang et al., 2008).

Os métodos que utilizam dados de mais de um terminal, em linhas com derivações, onde o método apresentado neste artigo se insere, são os apresentados pelas referências (Liu, 2008; Liao, 2008), os quais estão brevemente comentados a seguir.

O método desenvolvido em (Liu, 2008) aborda a localização de faltas em linhas de transmissão com circuitos duplos ou simples e com derivação. Este método baseia-se na medição dos fasores de tensão e corrente em todos os terminais da linha. Para a localização de faltas em linhas com vários terminais é aplicada a técnica de localização de faltas em redes de dois terminais para obter índices de localização de faltas, os quais são analisados através de um algoritmo para determinar o trecho de ocorrência da falta.

Em (Liao, 2008) é apresentado um método que utiliza técnicas baseadas na matriz de impedâncias da linha e nas medições de tensão em um ou em dois terminais na localização de faltas em linhas com circuito simples, não sendo necessárias medições de correntes. O algoritmo apresenta como resultado, para a localização de faltas em linhas com derivações, vários trechos possíveis de conter a falta, sendo necessário realizar uma intersecção dos trechos de ocorrência da falta obtidos pelo algoritmo para determinar o mais provável ou os mais prováveis trechos de ocorrência.

O método de localização de faltas proposto neste artigo utiliza dados de apenas dois terminais da linha e não necessita que sejam feitas análises posteriores nos resultados de distâncias de faltas, tais como análises e comparações de índices de localização de faltas ou intersecções entre locais possíveis de conter a falta.

Para mostrar a eficiência e a precisão do método de localização de faltas em determinar o trecho da linha, bem como a distância e a resistência de falta, foram feitas simulações com variação da distância, do tipo, da resistência e do local da falta no programa ATP (EMTP Center ,1987).

\section{MÉTODO DE LOCALIZAÇÃO DE FALTAS PROPOSTO}

O novo método de localização de faltas necessita dos seguintes dados para determinar as informações de falta, as quais são: trecho de ocorrência, distância e resistência:

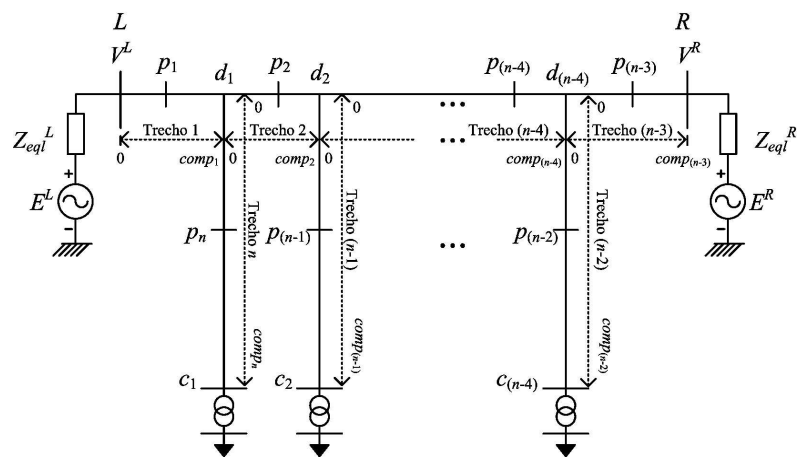

Figura 1: Linha com várias derivações e $n$ trechos

- Dados da linha de transmissão:

- Impedância série de sequência positiva em $(\Omega / \mathrm{km}): z l_{1}$

- Impedância série de sequência zero em $(\Omega / \mathrm{km}): z l_{0}$

- Admitância shunt de sequência positiva em $(\mathrm{S} / \mathrm{km}): y l_{1}$

- Admitância shunt de sequência zero em $(\mathrm{S} / \mathrm{km}): y l_{0}$

- Comprimento de cada trecho (Figura 1):comp

- Dados dos transformadores:

- Potência aparente nominal;

- Reatância de curto-circuito percentual. 
- Dados de tensão e corrente medidos nos terminais local $(L)$ e remoto $(R)$ :

- Tensões de fase pré-falta: $\left[V_{a b c}\right]_{\text {pré }}^{L}$ e $\left[V_{a b c}\right]_{\text {pré }}^{R}$

- Tensões de fase pós-falta: $\left[V_{a b c}\right]_{\text {pós }}^{L}$ e $\left[V_{a b c}\right]_{\text {pós }}^{R}$

- Correntes de fase pré-falta: $\left[I_{a b c}\right]_{\text {pré }}^{L}$ e $\left[I_{a b c}\right]_{\text {pré }}^{R}$

- Correntes de fase pós-falta: $\left[I_{a b c}\right]_{\text {pós }}^{L}$ e $\left[I_{a b c}\right]_{\text {pós }}^{R}$

- Referências de carga leve, média e pesada;

- Tipo de falta;

- Instante de ocorrência da falta.

Nos itens 2.1, 2.2 e 2.3 são apresentados os algoritmos e os procedimentos que integram o método de localização de faltas proposto neste artigo.

\subsection{Procedimento para cálculo dos equivalentes dos geradores}

Para estimar a impedância equivalente e a tensão interna dos geradores, são utilizadas as tensões e correntes pré-falta e pós-falta medidas nos terminais local e remoto.

Para realizar tal estimativa são calculadas as componentes simétricas das referidas tensões e correntes, como segue:

Para as tensões e correntes pré-falta tem-se:

$$
\begin{gathered}
{\left[V_{012}\right]_{\mathrm{pré}}^{L}=T^{-1}\left[V_{a b c}\right]_{\mathrm{pré}}^{L}} \\
{\left[V_{012}\right]_{\mathrm{pré}}^{R}=T^{-1}\left[V_{a b c}\right]_{\mathrm{pré}}^{R}} \\
{\left[I_{012}\right]_{\mathrm{pré}}^{L}=T^{-1}\left[I_{a b c}\right]_{\mathrm{pré}}^{L}} \\
{\left[I_{012}\right]_{\mathrm{pré}}^{R}=T^{-1}\left[I_{a b c}\right]_{\mathrm{pré}}^{R}}
\end{gathered}
$$

Para as tensões e correntes pós-falta tem-se:

$$
\begin{gathered}
{\left[V_{012}\right]_{\text {pós }}^{L}=T^{-1}\left[V_{a b c}\right]_{\text {pós }}^{L}} \\
{\left[V_{012}\right]_{\text {pós }}^{R}=T^{-1}\left[V_{a b c}\right]_{\text {pós }}^{R}} \\
{\left[I_{012}\right]_{\text {pós }}^{L}=T^{-1}\left[I_{a b c}\right]_{\text {pós }}^{L}}
\end{gathered}
$$

$$
\left[I_{012}\right]_{\mathrm{pós}}^{R}=T^{-1}\left[I_{a b c}\right]_{\mathrm{pós}}^{R}
$$

Onde $T$ é a matriz de transformação, dada por:

$$
\begin{gathered}
T=\left[\begin{array}{lll}
1 & 1 & 1 \\
1 & \alpha^{2} & \alpha \\
1 & \alpha & \alpha^{2}
\end{array}\right] \\
\alpha=1 \angle 120^{\circ} .
\end{gathered}
$$

Então, para as impedâncias equivalentes, de sequência positiva e zero, do gerador do terminal local, tem-se:

$$
\begin{aligned}
& Z_{1 e q l}^{L}=\left(\frac{V_{1 \text { pré }}^{L}-V_{1 \text { pós }}^{L}}{I_{1 \text { pós }}^{L}-I_{1 \mathrm{pré}}^{L}}\right) \\
& Z_{0 e q l}^{L}=\left(\frac{V_{0 \text { pré }}^{L}-V_{0 \text { pós }}^{L}}{I_{0 \text { pós }}^{L}-I_{0 \text { pré }}^{L}}\right)
\end{aligned}
$$

A tensão interna de sequência positiva do gerador do terminal local, o qual é considerado tendo somente esta sequência, é calculada da seguinte forma:

$$
E_{1}^{L}=Z_{1 e q l}^{L} I_{1 \mathrm{pré}}^{L}+V_{1 \mathrm{pré}}^{L}
$$

Para as impedâncias equivalentes, de sequência positiva e zero, do gerador do terminal remoto, tem-se:

$$
\begin{aligned}
& Z_{1 e q l}^{R}=\left(\frac{V_{1 \mathrm{prée}}^{R}-V_{1 \mathrm{pós}}^{R}}{I_{1 \mathrm{pós}}^{R}-I_{1 \mathrm{pré}}^{R}}\right) \\
& Z_{0 e q l}^{R}=\left(\frac{V_{0 \mathrm{pré}}^{R}-V_{0 \mathrm{pós}}^{R}}{I_{0 \mathrm{pós}}^{R}-I_{0 \mathrm{pré}}^{R}}\right)
\end{aligned}
$$

A tensão interna de sequência positiva do gerador do terminal remoto, o qual é considerado tendo somente esta sequência, é calculada da seguinte forma:

$$
E_{1}^{R}=Z_{1 e q l}^{R} I_{1 \mathrm{pré}}^{R}+V_{1 \mathrm{pré}}^{R}
$$

A impedância equivalente de sequência negativa, tanto para o terminal local quanto para o terminal remoto, é considerada idêntica à impedância equivalente de sequência positiva. 


\subsection{Algoritmo de estimativa das cargas}

O algoritmo de estimativa de cargas utilizado neste artigo é semelhante ao utilizado em (MANASSERO, 2006) e é composto por duas etapas. Na primeira etapa é feita uma estimativa inicial das cargas. Na segunda etapa é feita a estimativa final das cargas através da minimização da função objetivo, onde são utilizadas as estimativas iniciais das cargas como pontos de partida.

\section{A. Primeira etapa do algoritmo de estimativa das cargas}

Nesta etapa é realizada a primeira estimativa das cargas. O procedimento para realizar tal estimativa é descrito da seguinte forma:

Obtêm-se dos arquivos de planejamento da rede para estudos de fluxo de potência, as referências de carga leve, média e pesada.

Calcula-se, através de (16), a potência ativa total fornecida pela rede:

$$
P_{t o t}=R e\left\{\left[V_{a b c}\right]_{\mathrm{pré}}^{L}\left[I_{a b c}\right]_{\mathrm{pré}}^{L *}+\left[V_{a b c}\right]_{\mathrm{pré}}^{R}\left[I_{a b c}\right]_{\mathrm{pré}}^{R *}\right\}
$$

Calculada a potência ativa total fornecida pela linha, esta é comparada com as potências ativas das referências de carga leve, média e pesada.

Definida a referência a ser utilizada, é feita uma primeira estimativa da potência aparente das cargas distribuindo proporcionalmente a potência ativa total entre as mesmas.

É importante notar que, neste primeiro momento, apesar de a potência reativa de referência ter sido multiplicada pela razão entre a potência ativa total fornecida pela linha e a potência ativa total de referência das cargas, não se teve preocupação com o valor da potência reativa das mesmas.

Em (17) é descrita, matematicamente, a definição citada anteriormente:

$$
S_{\text {carga }}^{j}=\frac{P_{r}^{j}}{\sum_{j} P_{r}^{j}} P_{t o t}+j \frac{Q_{r}^{j}}{\sum_{j} P_{r}^{j}} P_{t o t}
$$

Onde:

$P_{r}^{j}$ é a potência ativa de referência para a carga $j$;

$Q_{r}^{j}$ é a potência reativa de referência para a carga $j$;

$\sum_{j} P_{r}^{j}$ é a somatória das potências ativas de referência das cargas.
Realizada a estimativa da potência aparente das cargas, é feita a estimativa das correntes nos trechos da rede utilizando-se as cargas estimadas e a $1^{a}$ lei de Kirchhoff.

Estimadas as correntes nos trechos da rede, estimam-se as perdas ativas na rede e nos transformadores, segundo as seguintes equações:

$$
P_{\text {trecho }}^{u}=3 \operatorname{Re}\left\{Z_{\text {trecho }}^{u}\right\}\left|I_{f}^{u}\right|^{2}
$$

$$
P_{\text {trafo }}^{j}=3 \operatorname{Re}\left\{Z_{\text {trafo }}^{j}\right\}\left|I_{f}^{j}\right|^{2}
$$

Onde:

$Z_{\text {trecho }}^{u}$ a impedância do trecho $u$;

$I_{f}^{u}$ é a corrente de fase do trecho $u$;

$Z_{\text {trafo }}^{j}$ é a impedância do transformador ligado à carga $j$ referida ao primário;

$I_{f}^{j}$ é a corrente de fase que passa na impedância do transformador.

Então as perdas totais são:

$$
P_{\text {perdas }}=\sum_{u} P_{\text {trecho }}^{u}+\sum_{j} P_{\text {trafo }}^{j}
$$

Subtraindo as perdas ativas da potência ativa total fornecida pela rede, obtêm-se uma estimativa da potência ativa total fornecida às cargas:

$$
P_{\text {totcargas }}=P_{\text {tot }}-P_{\text {perdas }}
$$

Então, estima-se novamente a potência aparente das cargas:

$$
S_{\text {carga }}^{j}=\frac{S_{r}^{j}}{\sum_{j} P_{r}^{j}} P_{\text {totcargas }}
$$

Estimadas as novas potências aparentes das cargas, calculam-se as impedâncias das cargas, obtendo uma estimativa inicial para ser usada na função objetivo. O cálculo da impedância das cargas é feito pela seguinte equação:

$$
Z_{\text {carga }}^{j}=\frac{\left|V_{\text {nominal }(\text { linha })}\right|^{2}}{S_{\text {carga }}^{j *}}
$$




\section{B. Segunda etapa do algoritmo de estimativa das cargas}

Feita a estimativa inicial das cargas, estas são utilizadas como entrada para a função objetivo, a qual executa os seguintes procedimentos:

- Calcula a matriz de admitâncias de sequência positiva da rede $\left[Y_{\text {barras }}\right]$, onde estão consideradas as impedâncias das cargas como variáveis;

- Com a matriz $\left[Y_{\text {barras }}\right]$ calcula a matriz de impedâncias de sequência positiva $\left[Z_{\text {barras }}\right]$;

- Calcula a tensão de sequência positiva nos nós da rede, $\left[V_{\text {barras }}\right]$, através da matriz $\left[Z_{\text {barras }}\right]$ e através das correntes de Norton de sequência positiva injetadas nos terminais local e remoto, as quais são calculadas através da tensão interna de sequência positiva dos geradores e de seus respectivos equivalentes de sequência positiva;

- Calcula as correntes de sequência positiva nos terminais local e remoto:

$$
\begin{aligned}
& I_{1 \text { précalc }}^{L}=\frac{\left(E_{1}^{L}-V_{1 \text { précalc }}^{L}\right)}{Z_{1 \text { eql }}^{L}} \\
& I_{1 \text { précalc }}^{R}=\frac{\left(E_{1}^{R}-V_{1 \text { précalc }}^{R}\right)}{Z_{1 \text { eql }}^{R}}
\end{aligned}
$$

Onde:

- $I_{1 \text { précalc }}^{L}$ e $I_{1 \text { précalc }}^{R}$ são as correntes de sequência positiva pré-falta, calculadas, nos terminais local e remoto;

- $E_{1}^{L}$ e $E_{1}^{R}$ são as tensões internas de sequência positiva dos geradores dos terminais local e remoto;

- $V_{1 \text { précalc }}^{L}$ e $V_{1 \text { précalc }}^{R}$ são as tensões de sequência positiva pré-falta, calculadas, nos terminais local e remoto;

$-Z_{1 e q l}^{L}$ e $Z_{1 e q l}^{R}$ são as impedâncias equivalentes de sequência positiva dos geradores dos terminais local e remoto.

-Calcula os erros relativos de tensão e corrente, ErroV e ErroI:

$$
\begin{gathered}
\text { ErroV }=\left|\frac{\left(V_{1 \text { prémed }}^{L}-V_{1 \text { précalc }}^{L}\right)}{V_{1 \text { prémed }^{L}}}\right|+ \\
\left|\frac{\left(V_{1 \text { prémed }}^{R}-V_{1 \text { précalc }}^{R}\right)}{V_{1 \text { prémed }}^{R}}\right|
\end{gathered}
$$

$$
\begin{gathered}
\text { ErroI }=\left|\frac{\left(I_{1 \text { prémed }}^{L}-I_{1 \text { précalc }}^{L}\right)}{I_{1 \text { prémed }}^{L}}\right|+ \\
\left|\frac{\left(I_{1 \text { prémed }}^{R}-I_{1 \text { précalc }}^{R}\right)}{I_{1 \text { prémed }}^{R}}\right|
\end{gathered}
$$

Onde:

- $I_{1 \text { prémed }}^{L}$ e $I_{1 \text { prémed }}^{R}$ são as correntes de sequência positiva pré-falta, medidas, nos terminais local e remoto;

- $V_{1 \text { prémed }}^{L}$ e $V_{1 \text { prémed }}^{R}$ são as tensões de sequência positiva pré-falta, medidas, nos terminais local e remoto.

- Minimiza a soma dos erros de tensão e corrente, $G$, para obter a estimativa das cargas:

$$
G=E r r o V+E r r o I
$$

\subsection{Algoritmo de localização de faltas}

O algoritmo de localização de faltas proposto neste trabalho realiza uma varredura nos trechos da rede para localizar a falta onde, conforme a figura 1 , cada trecho possuí início em zero. Como resultado, o algoritmo traz o trecho de ocorrência da falta, a distância de falta e a resistência de falta.

O algoritmo é composto por dois blocos, um que controla a varredura da rede e determina o trecho de ocorrência da falta e outro que determina as informações de falta, como a distância de falta e a resistência de falta.

\section{A. Processo de varredura da rede e obtenção do trecho de ocorrência da falta}

Com base na figura 1, a varredura da rede inicia-se no trecho 1 , onde um procedimento de minimização da função objetivo é utilizado dependendo do tipo de falta ocorrido, o qual está apresentado em detalhes no item $B$. A função objetivo é então minimizada variando-se a distância de falta dentro deste trecho e a resistência de falta, tendo como resultado da minimização o erro para o caso de falta localizada no trecho 1 , a distância de falta, que tem como referência o início deste trecho, e a resistência de falta.

Obtida as informações para o trecho 1, passa-se para o trecho 2 e da mesma forma como para o trecho 1 , a função objetivo é minimizada e retorna o erro de a falta estar no trecho 2 , a distância de falta, que tem como referência o início deste trecho, e a resistência de falta. 


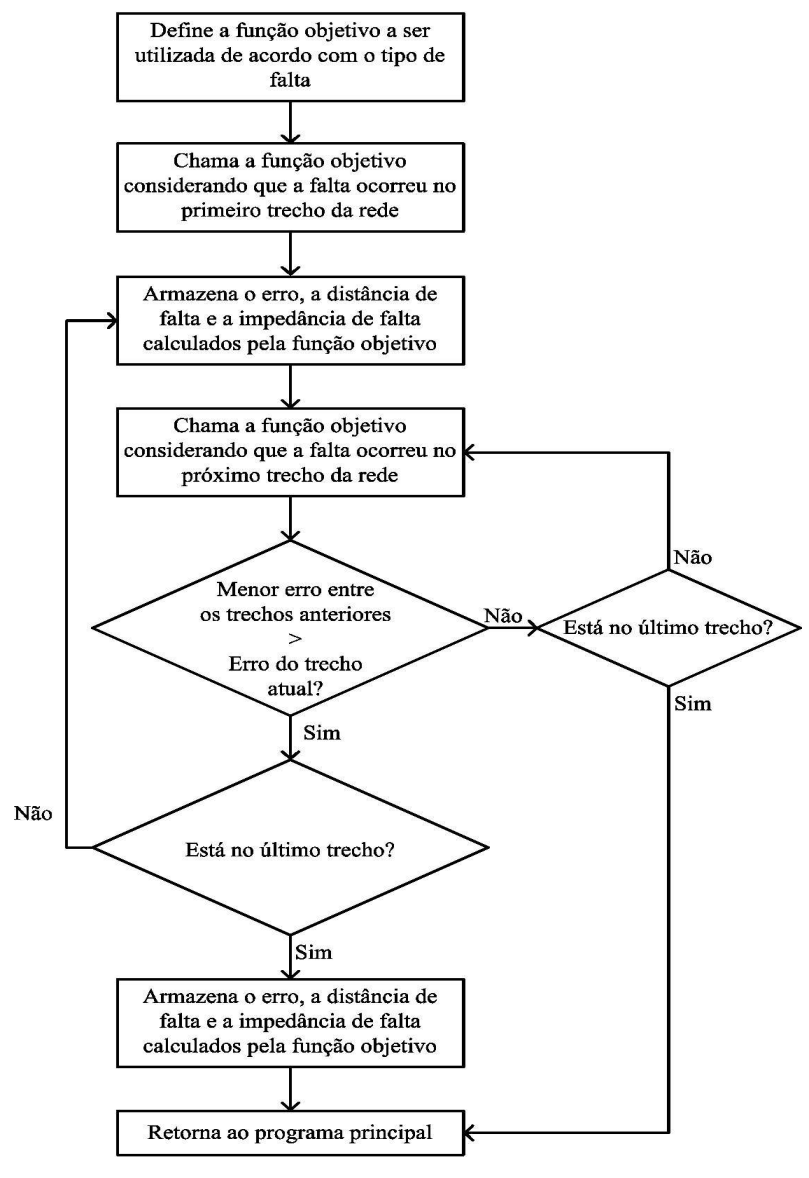

Figura 2: Fluxograma do processo de varredura da rede

Os erros obtidos para o trecho 1 e para o trecho 2 são, então, comparados, sendo o trecho com maior erro descartado. O processo de varredura é realizado até que o último trecho seja analisado.

Ao terminar o processo de varredura, o trecho de ocorrência da falta, a distância de falta e a resistência de falta são conhecidos e disponibilizados pelo método de localização de faltas.

O fluxograma da figura 2 apresenta o processo de varredura da rede.

\section{B. Processo de determinação das informações de falta}

Para obter o erro de a falta estar em um determinado trecho, a distância e a resistência de falta, foi desenvolvida, para cada tipo de falta, uma função objetivo.

De uma maneira geral, utilizando a rede da figura 1 como exemplo e que o processo de varredura descrito no item 2.3-A esteja no trecho 1 , o processo de minimização da função objetivo pode ser descrito da seguinte forma:

A figura 3 mostra o fluxograma para minimização da função objetivo.

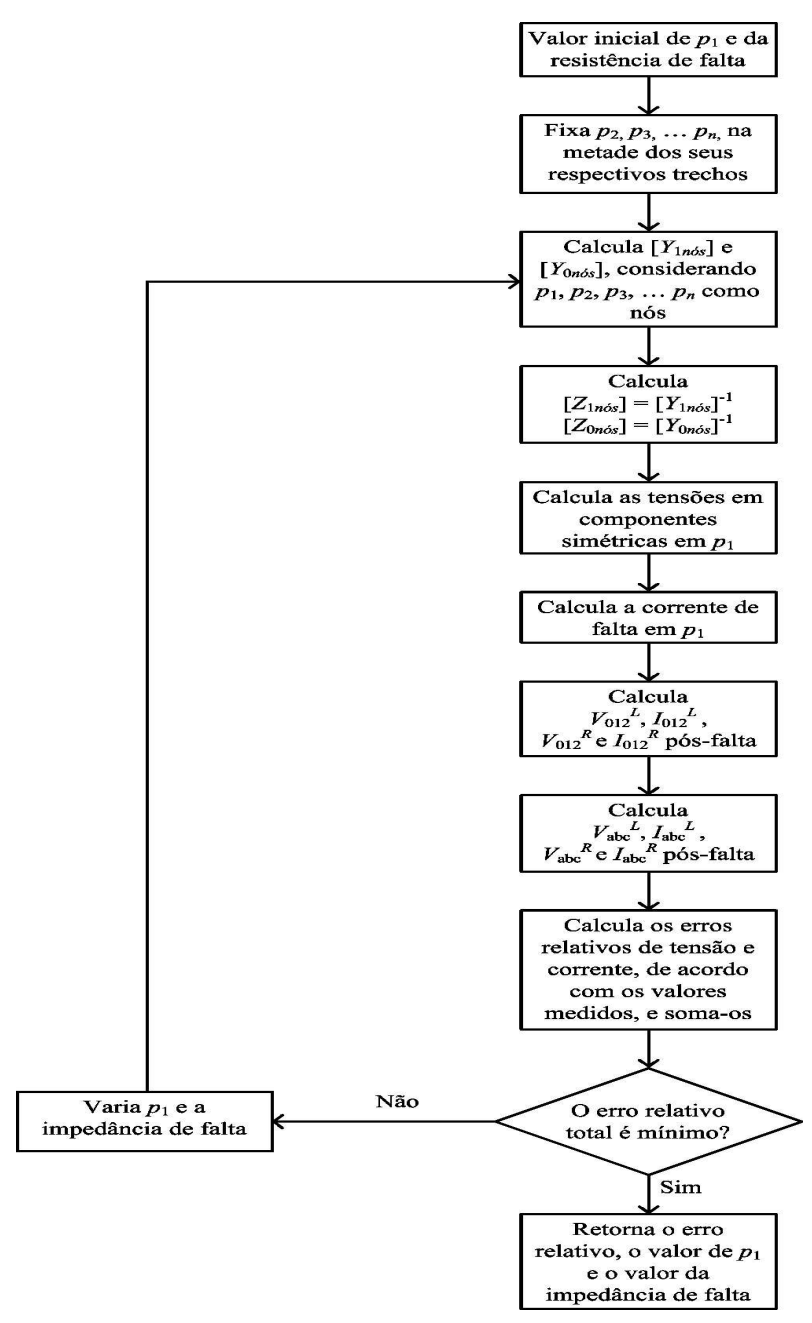

Figura 3: Fluxograma para minimização da função objetivo

1. Especifica-se um valor inicial para o ponto $p_{1}$ e para a resistência de falta;

2. Fixa-se os pontos $p_{2}, p_{3}, \ldots, p_{n}$ na metade dos seus respectivos trechos, ou seja, $\operatorname{comp}_{2} / 2, \operatorname{comp}_{3} / 2, \ldots$, $\operatorname{comp}_{n} / 2$;

3. Calculam-se as matrizes de admitâncias e impedâncias (através da inversa da matriz de admitâncias) de sequência positiva e zero (no caso de faltas que 
envolvam a terra) considerando, além das barras dos terminais local e remoto, dos pontos de derivação $\left(d_{1}\right.$ a $\left.d_{(n-5)}\right)$ e das barras das cargas $\left(c_{1}\right.$ a $\left.c_{(n-5)}\right)$, os pontos $p_{1}$ a $p_{n}$ como nós nas matrizes;

4. Calcula-se a tensão de sequência positiva, pré-falta, nos nós definidos no passo 3 através da corrente de Norton de sequência positiva injetada nos terminais local e remoto, a qual é calculada através da tensão interna dos geradores e de seus respectivos equivalentes, e da matriz de impedâncias de sequência positiva;

5. Das tensões obtidas no passo 4 considera-se somente a tensão no nó correspondente ao ponto $p_{1}$;

6. Calcula-se a corrente de falta no ponto $p_{1}$ através da tensão de sequência positiva obtida no passo 5 , através das impedâncias equivalentes de Thévenin de sequências positiva, negativa (considerada tendo o mesmo valor da impedância de sequência positiva) e zero vistas pelo ponto $p_{1}$ e pela resistência de falta, sendo que as impedâncias equivalentes de Thévenin são extraídas das matrizes de sequência positiva e zero. $\mathrm{O}$ cálculo da corrente de falta é dependente do tipo de falta, podendo uma ou outra impedância equivalente de Thévenin de sequência positiva, negativa e zero serem utilizadas no cálculo ou não;

7. Obtida a corrente de falta, calculam-se, utilizando também as matrizes de sequências positiva e zero (para o caso de faltas que envolvam esta componente) e as correntes de Norton injetadas pelos geradores equivalentes, as tensões em componentes simétricas pós-falta em todos os nós da rede;

8. Das tensões obtidas no passo 7 considera-se somente as tensões obtidas para os terminais local e remoto;

9. Calculam-se as correntes de sequência positiva, negativa e zero (no caso de falta envolvendo a terra) nos terminais local e remoto, através das tensões em componentes simétricas pós-falta nestes terminais, obtidas no passo 8, e através das impedâncias equivalentes de sequência positiva, negativa (considerada tendo o mesmo valor da impedância de sequência positiva) e zero (para faltas que envolvem a terra) dos geradores;

10. Realiza-se a transformação das tensões e correntes em componentes simétricas pós-falta obtidas nos passos $8 \mathrm{e}$ 9 para tensões e correntes de fase pós-falta;

11. Calcula-se o erro relativo entre as tensões pós-falta medidas e as calculadas no passo 10, dos terminais local e remoto;
12. Calcula-se o erro relativo entre as correntes pós-falta medidas e as calculadas no passo 10 , dos terminais local e remoto;

13. Somam-se os erros relativos das tensões com os da corrente, obtendo um erro relativo total;

14. Minimiza-se o erro relativo total, obtido no passo 13, variando-se o ponto $p_{1}$, ao longo do trecho 1 , e a resistência de falta.

As matrizes de impedâncias de sequência positiva e zero, as quais são, respectivamente, o inverso das matrizes de admitâncias de sequência positiva e zero, da rede da figura 1, possuem a seguinte forma:

$$
\begin{aligned}
{\left[Z_{\text {1nós }}\right]=} & {\left[\begin{array}{lllll}
Z_{1}^{L L} & Z_{1}^{L p_{1}} & Z_{1}^{L d_{1}} & \cdots & Z_{1}^{L c_{1}} \\
Z_{1}^{p_{1} L} & Z_{1}^{p_{1} p_{1}} & Z_{1}^{p_{1} d_{1}} & \cdots & Z_{1}^{p_{1} c_{1}} \\
Z_{1}^{d_{1} L} & Z_{1}^{d_{1} p_{1}} & Z_{1}^{d_{1} d_{1}} & \cdots & Z_{1}^{d_{1} c_{1}} \\
\vdots & \vdots & \vdots & \ddots & \vdots \\
Z_{1}^{c_{1} L} & Z_{1}^{c_{1} p_{1}} & Z_{1}^{c_{1} d_{1}} & \cdots & Z_{1}^{c_{1} c_{1}}
\end{array}\right] } \\
{\left[Z_{\text {0nós }}\right]=} & {\left[\begin{array}{llllll}
Z_{0}^{L L} & Z_{0}^{L p_{1}} & Z_{0}^{L d_{1}} & \cdots & Z_{0}^{L c_{1}} \\
Z_{0}^{p_{1} L} & Z_{0}^{p_{1} p_{1}} & Z_{0}^{p_{1} d_{1}} & \cdots & Z_{0}^{p_{1} c_{1}} \\
Z_{0}^{d_{1} L} & Z_{0}^{d_{1} p_{1}} & Z_{0}^{d_{1} d_{1}} & \cdots & Z_{0}^{d_{1} c_{1}} \\
\vdots & \vdots & \vdots & \ddots & \vdots \\
Z_{0}^{c_{1} L} & Z_{0}^{c_{1} p_{1}} & Z_{0}^{c_{1} d_{1}} & \cdots & Z_{0}^{c_{1} c_{1}}
\end{array}\right] }
\end{aligned}
$$

As diagonais das matrizes (29) e (30) são as impedâncias vistas pelos nós da rede da figura 1 .

As correntes de falta são determinadas da seguinte maneira (considerando o nó $p_{1}$ ):

- Falta fase A-terra:

$$
I_{0}^{\text {falta }}=I_{1}^{\text {falta }}=I_{2}^{\text {falta }}=\frac{V_{1 \text { précalc }}^{p_{1}}}{2 Z_{1}^{p_{1} p_{1}}+Z_{0}^{p_{1} p_{1}}+3 R_{f}}
$$

- Falta fases BC-terra:

$$
\begin{gathered}
I_{0}^{f a l t a}=\frac{-y_{0}^{f} y_{1}^{f} V_{1 \text { précalc }}^{p_{1}}}{2 y_{1}^{f}+y_{0}^{f}} \\
I_{1}^{\text {falta }}=\frac{\left(y_{0}^{f}+y_{1}^{f}\right) y_{1}^{f} V_{1 \text { précalc }}^{p_{1}}}{2 y_{1}^{f}+y_{0}^{f}}
\end{gathered}
$$




$$
I_{2}^{f a l t a}=\frac{-y_{1}^{f 2} V_{1 \text { précalc }}^{p_{1}}}{2 y_{1}^{f}+y_{0}^{f}}
$$

Onde:

$$
y_{0}^{f}=\frac{1}{Z_{0}^{p_{1} p_{1}}+R_{f}} \text { e } y_{1}^{f}=\frac{1}{Z_{1}^{p_{1} p_{1}}+R_{f}}
$$

- Falta fases BC:

$$
I_{1}^{\text {falta }}=-I_{2}^{\text {falta }}=\frac{V_{1 \text { précalc }}^{p_{1}}}{2 Z_{1}^{p_{1} p_{1}}+R_{f}} \text { e } I_{0}^{f a l t a}=0
$$

- Falta fases ABC:

$$
I_{1}^{\text {falta }}=\frac{V_{1 \text { précalc }}^{p_{1}}}{Z_{1}^{p_{1} p_{1}}+R_{f}} \text { e } I_{0}^{\text {falta }}=I_{2}^{\text {falta }}=0
$$

As tensões e correntes em componentes simétricas pós-falta são calculadas da seguinte forma:

Para as tensões nos nós:

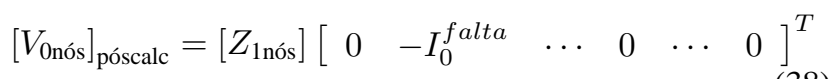

$$
\left[V_{1 \text { nós }}\right]_{\text {póscalc }}=\left[Z_{1 \text { nós }}\right]\left[\begin{array}{llll}
I_{1}^{N L} & -I_{1}^{\text {falta }} & \cdots I_{1}^{N R} \cdots 0
\end{array}\right]_{(39)}^{T}
$$

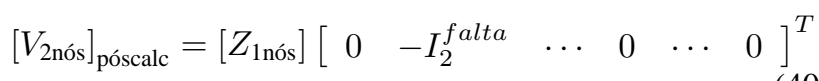

Para as correntes no terminal local:

$$
\begin{gathered}
I_{\text {Opóscalc }}^{L}=\frac{-V_{\text {Opóscalc }}^{L}}{Z_{0 e q l}^{L}} \\
I_{1 \text { póscalc }}^{L}=I_{1}^{N L}-\frac{V_{1 \text { póscalc }}^{L}}{Z_{1 e q l}^{L}} \\
I_{2 \text { póscalc }}^{L}=\frac{-V_{2 \text { póscalc }}^{L}}{Z_{1 e q l}^{L}}
\end{gathered}
$$

Para as correntes no terminal remoto:

$$
I_{\text {Opóscalc }}^{R}=\frac{-V_{\text {0póscalc }}^{R}}{Z_{0 e q l}^{R}}
$$

$$
\begin{gathered}
I_{1 \mathrm{póscalc}}^{R}=I_{1}^{N R}-\frac{V_{1 \text { póscalc }}^{R}}{Z_{1 \text { eql }}^{R}} \\
I_{2 \text { póscalc }}^{R}=\frac{-V_{2 \text { póscalc }}^{R}}{Z_{1 e q l}^{R}}
\end{gathered}
$$

\section{RESULTADOS}

Para analisar o desempenho do método de localização de faltas proposto neste artigo, foram feitas várias simulações de faltas através do programa ATP, o qual após a simulação gera um arquivo de dados equivalente a uma oscilografia digital. Os dados são, então, utilizados em uma rotina de pós-processamento, implementada no programa MATLAB (MathWorks, 1992), que realiza a filtragem digital dos mesmos, através de um filtro digital baseado no método dos mínimos quadrados em série com um filtro digital passa-baixas de Butterworth, para a obtenção dos fasores a $60 \mathrm{~Hz}$ das tensões e correntes pré-falta e pós-falta.

O desempenho do método de localização de faltas será analisado com base na variação de uma série de fatores, tais como:

- Variação da distância de falta em cada trecho da rede;

- Faltas próximas aos pontos de derivação;

- Variação do tipo de falta;

- Variação da resistência de falta.

- A rede utilizada nas simulações é apresentada na figura 4, onde sua estrutura é descrita a seguir:Linha de transmissão:

- Tensão de operação: 138 kV;

- Continuamente transposta em todos os trechos;

- Circuito simples;

- Comprimento dos trechos:

Trecho 1: $30 \mathrm{~km}$;

Trecho 2: $40 \mathrm{~km}$;

Trecho 3: $30 \mathrm{~km}$;

Trecho 4: $70 \mathrm{~km}$;

Trecho 5: $30 \mathrm{~km}$;

Trecho 6: $40 \mathrm{~km}$;

Trecho 7: $40 \mathrm{~km}$. 
-Parâmetros (todos os trechos possuem mesmos parâmetros):

$$
\begin{aligned}
& z l_{1}=0,183+\mathrm{j} 0,495 \Omega / \mathrm{km} ; \\
& y l_{1}=\mathrm{j} 3,316 \mu \mathrm{S} / \mathrm{km} ; \\
& z l_{0}=0,465+\mathrm{j} 1,841 \Omega / \mathrm{km} ; \\
& y l_{0}=\mathrm{j} 1,890 \mu \mathrm{S} / \mathrm{km} .
\end{aligned}
$$

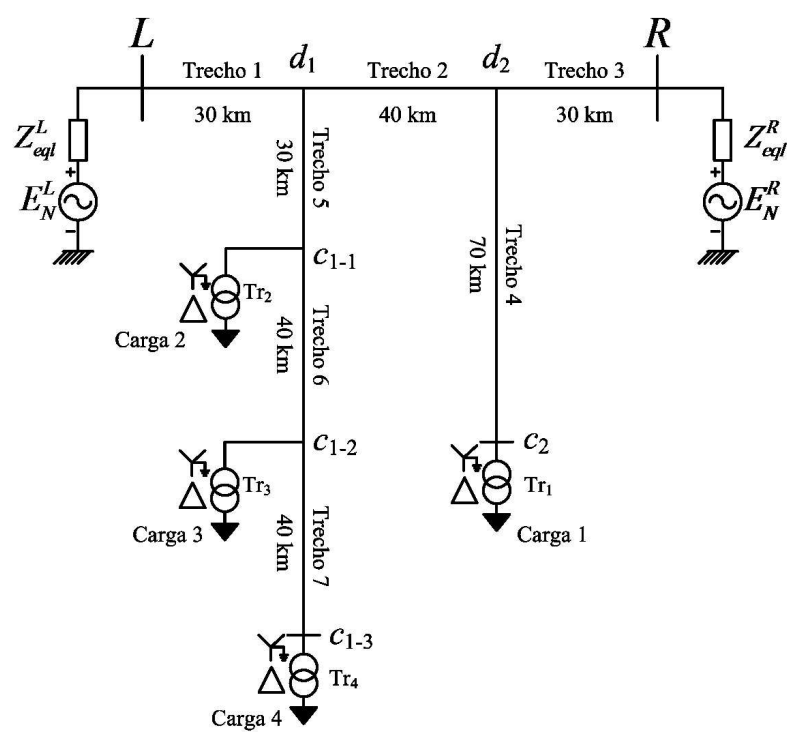

Figura 4: Rede simulada para avaliação do método de localização de faltas proposto

- Transformadores:

- Tr1: Ligação Estrela aterrada/delta, 30 MVA;

- Tr2: Ligação Estrela aterrada/delta, 30 MVA;

- Tr3: Ligação Estrela aterrada/delta, 30 MVA;

- Tr4: Ligação Estrela aterrada/delta, 30 MVA.

Todos possuem $x_{c c}=10 \%$.

- Geradores:

- Terminal local:

Potência de curto-circuito trifásico:

$$
0,97 \angle 84,14^{\circ} \mathrm{GVA} \text {; }
$$

Potência de curto-circuito fase-terra:

$$
0,72 \angle 83,46^{\circ} \mathrm{GVA} \text {; }
$$

Impedância equivalente de sequência positiva:

$$
Z_{1 e q l}^{L}=2+j 19,5 \Omega
$$

Impedância equivalente de sequência zero:

$$
Z_{0 e q l}^{L}=5+j 39,5 \Omega
$$

- Terminal remoto:

Potência de curto-circuito trifásico:

$$
2,23 \angle 83,29^{\circ} \mathrm{GVA} \text {; }
$$

Potência de curto-circuito fase-terra:

$$
1,60 \angle 83,57^{\circ} \mathrm{GVA} \text {; }
$$

Impedância equivalente de sequência positiva:

$$
Z_{1 e q l}^{L}=1+j 8,5 \Omega
$$

Impedância equivalente de sequência zero:

$$
Z_{0 e q l}^{L}=2+j 18,5 \Omega
$$

\section{- Cargas:}

- Carga 1: 10 $\angle 36,87^{\circ} \mathrm{MVA}$;

- Carga 2: $7 \angle 23,07^{\circ} \mathrm{MVA}$;

- Carga 3: 7,94 $\angle 18,19^{\circ} \mathrm{MVA}$;

- Carga 4: 12,7 $\angle 25,84^{\circ}$ MVA.

\subsection{Variação da distância de falta}

Neste item estão apresentados os resultados da análise de desempenho do algoritmo proposto para a variação da distância de falta, conforme as seguintes definições:

Para as tabelas 1 e 3 , conforme figura 4:

- As distâncias de falta de todos os trechos são medidas em relação ao terminal local $(L)$;

- Os erros relativos obtidos para os trechos 1, 2 e 3 foram calculados em relação ao comprimento da linha principal, ou seja, $100 \mathrm{~km}$, para o trecho 4 foram calculados em relação a distância entre o terminal local e o final da derivação $2\left(c_{2}\right)$, ou seja, $140 \mathrm{~km}$ e para os trechos 5, 6 e 7 foram calculados em relação a distância entre o terminal local e o final da derivação $1\left(c_{1-3}\right)$, ou seja, $140 \mathrm{~km}$.

Para as tabelas 2 e 4, conforme figura 4:

- As distâncias de falta para os trechos 1, 2 e 3 são medidas em relação ao terminal local $(L)$. Para os trechos 4 e 5 são medidas em relação ao inicio da derivação $2\left(d_{2}\right)$ e ao inicio da derivação $1\left(d_{1}\right)$, respectivamente; 
- Os erros relativos obtidos para os trechos 1, 2 e 3 foram calculados em relação ao comprimento da linha principal, ou seja, $100 \mathrm{~km}$. Para os trechos 4 e 5 foram calculados em relação a distância entre o terminal local e o final da derivação $2\left(c_{2}\right)$, ou seja, $140 \mathrm{~km}$ e em relação a distância entre o terminal local e o final da derivação $1\left(c_{1-3}\right)$, ou seja, $140 \mathrm{~km}$, respectivamente.

A seguir estão apresentados os resultados da simulação para:

- Falta Fase A - terra;

- Resistência de falta igual a $5 \Omega$;

- Cargas e equivalentes estimados;

Tabela 1: Resultados para a variação da distância de falta para faltas fase A-terra

\begin{tabular}{|c|c|c|c|c|c|}
\hline Trecho & $\begin{array}{l}\text { Distância } \\
\text { simulada } \\
(\mathrm{km})\end{array}$ & $\begin{array}{l}\text { Distância } \\
\text { calculada } \\
(\mathrm{km})\end{array}$ & $\begin{array}{l}\text { Erro } \\
\text { absoluto } \\
\text { (m) }\end{array}$ & $\begin{array}{l}\text { Erro } \\
\text { relativo } \\
(\%)\end{array}$ & $\begin{array}{l}\text { Resistência } \\
\text { calculada } \\
(\Omega)\end{array}$ \\
\hline \multirow{3}{*}{1} & 5 & 5,00 & 0 & 0 & 5,00 \\
\hline & 15 & 15,00 & 0 & 0 & 5,00 \\
\hline & 25 & 24,96 & 40 & $-0,040$ & 4,99 \\
\hline \multirow{3}{*}{2} & 35 & 35,02 & 20 & 0,020 & 5,00 \\
\hline & 50 & 50,04 & 40 & 0,040 & 4,98 \\
\hline & 60 & 59,96 & 40 & $-0,040$ & 4,99 \\
\hline \multirow{3}{*}{3} & 75 & 75,00 & 0 & 0 & 5,00 \\
\hline & 85 & 85,00 & 0 & 0 & 5,00 \\
\hline & 95 & 95,00 & 0 & 0 & 5,00 \\
\hline \multirow{3}{*}{4} & 80 & 79,98 & 20 & $-0,014$ & 5,01 \\
\hline & 105 & 104,98 & 20 & $-0,014$ & 5,01 \\
\hline & 130 & 129,97 & 30 & $-0,021$ & 4,99 \\
\hline \multirow{3}{*}{5} & 35 & 34,99 & 10 & $-0,007$ & 4,99 \\
\hline & 45 & 44,99 & 10 & $-0,007$ & 5,00 \\
\hline & 55 & 55,00 & 0 & 0 & 5,02 \\
\hline \multirow{3}{*}{6} & 65 & 64,95 & 50 & $-0,036$ & 5,02 \\
\hline & 80 & 79,96 & 40 & $-0,029$ & 5,05 \\
\hline & 90 & 89,95 & 50 & $-0,036$ & 4,99 \\
\hline \multirow{3}{*}{7} & 105 & 105,07 & 70 & 0,050 & 5,03 \\
\hline & 120 & 119,95 & 50 & $-0,036$ & 5,10 \\
\hline & 130 & 129,97 & 30 & $-0,021$ & 5,04 \\
\hline
\end{tabular}

- Falta trifásica;

- Resistência de falta igual a $5 \Omega$.

- Cargas e equivalentes estimados.

Como mostram as tabelas 2 e 4, qualquer um dos trechos envolvidos na falta podem ser utilizados como resultado, mas como o algoritmo retorna apenas uma saída, ele irá escolher o resultado que contiver o menor erro.
Tabela 2: Resultados para falta fase $\mathrm{A}$ - terra nos pontos de derivação $d_{1}$ e $d_{2}$

\begin{tabular}{|l|l|l|l|l|l|l|}
\hline Ponto & $\begin{array}{l}\text { Trechos } \\
\text { envolvidos }\end{array}$ & $\begin{array}{l}\text { Distância } \\
\text { simulada } \\
(\mathrm{km})\end{array}$ & $\begin{array}{l}\text { Distância } \\
\text { calculada } \\
(\mathrm{km})\end{array}$ & $\begin{array}{l}\text { Erro } \\
\text { absoluto } \\
(\mathrm{m})\end{array}$ & $\begin{array}{l}\text { Erro } \\
\text { relativo } \\
(\%)\end{array}$ & $\begin{array}{l}\text { Resistencia } \\
\text { calculada } \\
(\Omega)\end{array}$ \\
\hline \multirow{3}{*}{$d_{1}$} & 1 & 30 & 30,00 & 0 & 0 & 5,02 \\
\cline { 2 - 7 } & 2 & 0 & 0 & 0 & 0 & 5,01 \\
\cline { 2 - 7 } & 5 & 0 & 0 & 0 & 0 & 5,01 \\
\hline \multirow{3}{*}{$d_{2}$} & 2 & 70 & 70,00 & 0 & 0 & 4,99 \\
\cline { 2 - 7 } & 3 & 0 & 0 & 0 & 0 & 5,00 \\
\cline { 2 - 7 } & 4 & 0 & 0 & 0 & 0 & 5,00 \\
\hline
\end{tabular}

Dentre todas as simulações de variação da distância de falta, o erro relativo máximo, em módulo, obtido foi de $0,05 \%$, evidenciando a precisão do método frente a este fator.

\subsection{Variação do tipo de falta}

Neste item estão apresentados os resultados da análise de desempenho do algoritmo proposto para a variação do tipo de falta, onde as definições descritas no item 3.1 são válidas para este item.

A seguir estão apresentados os resultados da simulação para:

- Distância de falta igual a $50 \mathrm{~km}$ - Trecho 2;

- Resistência de falta igual a $5 \Omega$;

- Cargas e equivalentes estimados.

- Distância de falta igual a $130 \mathrm{~km}$ - Trecho 4;

- Resistência de falta igual a $5 \Omega$;

- Cargas e equivalentes estimados.

- Distância de falta igual a $80 \mathrm{~km}$ - Trecho 6;

- Resistência de falta igual a $5 \Omega$;

- Cargas e equivalentes estimados.

Dentre todas as simulações de variação do tipo de falta, o erro relativo máximo, em módulo, obtido foi de $0,1 \%$, evidenciando a precisão do método frente a este fator.

\subsection{Variação da resistência de falta}

Neste item estão apresentados os resultados da análise de desempenho do algoritmo proposto para a variação da 
Tabela 3: Resultados para a variação da distância de falta para faltas trifásicas

\begin{tabular}{|l|l|l|l|l|l|}
\hline Trecho & $\begin{array}{l}\text { Distância } \\
\text { simulada } \\
(\mathrm{km})\end{array}$ & $\begin{array}{l}\text { Distância } \\
\text { calculada } \\
(\mathrm{km})\end{array}$ & $\begin{array}{l}\text { Erro } \\
\text { absoluto } \\
(\mathrm{m})\end{array}$ & $\begin{array}{l}\text { Erro } \\
\text { relativo } \\
(\%)\end{array}$ & $\begin{array}{l}\text { Resistencia } \\
\text { calculada } \\
(\Omega)\end{array}$ \\
\hline \multirow{4}{*}{1} & 5 & 5,00 & 0 & 0 & 5,00 \\
\cline { 2 - 6 } & 15 & 15,00 & 0 & 0 & 5,00 \\
\cline { 2 - 6 } & 25 & 25,01 & 10 & 0,010 & 4,99 \\
\hline \multirow{4}{*}{2} & 35 & 34,98 & 20 & $-0,020$ & 5,00 \\
\cline { 2 - 6 } & 50 & 50,02 & 20 & 0,020 & 5,00 \\
\cline { 2 - 6 } & 60 & 59,98 & 20 & $-0,020$ & 5,00 \\
\hline \multirow{4}{*}{3} & 75 & 75,00 & 0 & 0 & 5,00 \\
\cline { 2 - 6 } & 85 & 85,00 & 0 & 0 & 5,00 \\
\cline { 2 - 6 } & 95 & 95,00 & 0 & 0 & 5,00 \\
\hline \multirow{4}{*}{5} & 80 & 79,99 & 10 & $-0,007$ & 5,00 \\
\cline { 2 - 6 } & 105 & 105,00 & 0 & 0 & 5,00 \\
\cline { 2 - 6 } & 130 & 129,99 & 10 & $-0,007$ & 4,99 \\
\cline { 2 - 6 } & 35 & 34,99 & 10 & $-0,007$ & 5,00 \\
\cline { 2 - 6 } & 45 & 44,99 & 10 & $-0,007$ & 5,00 \\
\hline \multirow{4}{*}{6} & 55 & 54,97 & 30 & $-0,021$ & 5,01 \\
\cline { 2 - 6 } & 65 & 64,98 & 20 & $-0,014$ & 5,00 \\
\hline & 80 & 80,06 & 60 & 0,043 & 5,00 \\
\cline { 2 - 6 } & 90 & 89,98 & 20 & $-0,014$ & 5,01 \\
\hline \multirow{4}{*}{7} & 105 & 104,96 & 40 & $-0,029$ & 5,00 \\
\cline { 2 - 6 } & 120 & 120,04 & 40 & 0,029 & 4,98 \\
\hline
\end{tabular}

resistência de falta, onde as definições descritas no item 3.1 são válidas para este item.

A seguir estão apresentados os resultados da simulação para:

\section{- Distância de falta igual a 50 km - Trecho 2;}

\section{- Cargas e equivalentes estimados.}

- Distância de falta igual a 130 km - Trecho 4;

\section{- Cargas e equivalentes estimados.}

\section{- Distância de falta igual a 80 km - Trecho 6;}

\section{- Cargas e equivalentes estimados.}

figure

Considerando-se todas as simulações de variação da resistência de falta, o erro relativo máximo, em módulo, obtido foi de $0,27 \%$, mostrando que a precisão do método de localização de faltas proposto é insensível a faltas de alta resistência.
Tabela 4: Resultados para falta fases $A B C$ nos pontos de derivação $d_{1}$ e $d_{2}$

\begin{tabular}{|c|c|c|c|c|c|c|}
\hline Ponto & $\begin{array}{l}\text { Trechos } \\
\text { envolvios }\end{array}$ & $\begin{array}{c}\text { Distância } \\
\text { simulada } \\
(\mathrm{kml})\end{array}$ & $\begin{array}{l}\text { Distrância } \\
\text { calculada } \\
(\mathrm{km})\end{array}$ & $\begin{array}{l}\text { Erro } \\
\text { absoluto } \\
\text { (m) }\end{array}$ & $\begin{array}{l}\text { Erro } \\
\text { relativo } \\
(\%)\end{array}$ & 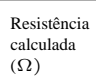 \\
\hline \multirow{3}{*}{$d_{1}$} & 1 & 30 & 29,99 & 10 & $-0,010$ & 5,00 \\
\hline & 2 & 0 & 0,01 & 10 & 0,010 & 4,99 \\
\hline & 5 & 0 & 0 & 0 & 0 & 5,00 \\
\hline \multirow{3}{*}{$d_{2}$} & 2 & 40 & 40,00 & 0 & 0 & 5,00 \\
\hline & 3 & 0 & 0 & 0 & 0 & 5,00 \\
\hline & 4 & 0 & 0 & 0 & 0 & 5,00 \\
\hline
\end{tabular}

Tabela 5: Resultados para a variação do tipo de falta para falta no trecho 2 a $50 \mathrm{~km}$ do terminal local $(\boldsymbol{L})$

\begin{tabular}{|l|l|l|l|l|}
\hline Tipo de fata & $\begin{array}{l}\text { Distância } \\
\text { calculalda (km) }\end{array}$ & Erro absoluto $(\mathrm{m})$ & $\begin{array}{l}\text { Erro } \\
(\%)\end{array}$ & $\begin{array}{l}\text { Relative } \\
(\Omega)\end{array}$ \\
\hline AN & 50,04 & 40 & 0,040 & 4,98 \\
\hline BN & 50,02 & 20 & 0,020 & 4,99 \\
\hline CN & 49,99 & 10 & $-0,010$ & 4,99 \\
\hline ABN & 49,99 & 10 & $-0,010$ & 5,00 \\
\hline BCN & 50,01 & 10 & 0,010 & 4,99 \\
\hline CAN & 50,02 & 20 & 0,020 & 5,00 \\
\hline AB & 50,04 & 40 & 0,040 & 4,98 \\
\hline BC & 50,00 & 0 & 0 & 5,00 \\
\hline CA & 50,03 & 30 & 0,030 & 5,00 \\
\hline ABC & 50,02 & 20 & 0,020 & 5,00 \\
\hline
\end{tabular}

\section{CONCLUSÕES}

Neste artigo foi proposto um novo método de localização de faltas em linhas de transmissão transpostas e com múltiplas derivações utilizando apenas os dados de tensão e corrente, pré-falta e pós-falta, obtidos nos terminais local e remoto onde se localizam as subestações.

Os resultados apresentados, obtidos a partir de simulações de faltas no programa ATP, mostram a eficiência e precisão do método proposto na obtenção das informações de falta: trecho de ocorrência, distância e resistência, frente a variações da distância, do tipo e resistência de falta, sendo que em todos os casos de simulação os equivalentes dos geradores foram calculados pelo procedimento descrito no item $2.1 \mathrm{e}$ as cargas foram estimadas pelo procedimento descrito no item 2.2.

A aplicação desse método a linhas com circuitos paralelos pode ser feita introduzindo-se na matriz de admitâncias de sequência zero a admitância mútua (inverso da reatância mútua) existente entre os circuitos de sequência zero.

Para trechos de linha com geração no final, como por exemplo no caso de usinas térmicas ou pequenas centrais hidrelétricas, conectadas à rede básica através de 
Tabela 6: Resultados para a variação do tipo de falta para falta no trecho 4 a $130 \mathrm{~km}$ do terminal local $(\boldsymbol{L})$

\begin{tabular}{|l|l|l|l|l|}
\hline Tipo de falta & $\begin{array}{l}\text { Distância } \\
\text { calculada }(\mathrm{km})\end{array}$ & Erro absoluto $(\mathrm{m})$ & $\begin{array}{l}\text { Erro } \\
(\%)\end{array}$ & $\begin{array}{l}\text { relativo } \\
(\Omega)\end{array}$ \\
\hline $\mathrm{AN}$ & 129,96 & 40 & $-0,029$ & 5,06 \\
\hline $\mathrm{BN}$ & 129,87 & 130 & $-0,093$ & 5,02 \\
\hline $\mathrm{CN}$ & 129,96 & 40 & $-0,029$ & 4,96 \\
\hline $\mathrm{ABN}$ & 129,93 & 70 & $-0,050$ & 5,03 \\
\hline $\mathrm{BCN}$ & 129,91 & 90 & $-0,064$ & 5,01 \\
\hline $\mathrm{CAN}$ & 129,97 & 30 & $-0,021$ & 5,00 \\
\hline $\mathrm{AB}$ & 129,93 & 70 & $-0,050$ & 5,06 \\
\hline $\mathrm{BC}$ & 129,93 & 30 & $-0,021$ & 4,99 \\
\hline $\mathrm{CA}$ & 129,98 & 20 & $-0,014$ & 5,02 \\
\hline ABC & 129,96 & 40 & $-0,029$ & 5,01 \\
\hline
\end{tabular}

Tabela 7: Resultados para a variação do tipo de falta para falta no trecho 6 a $80 \mathrm{~km}$ do terminal local $(\boldsymbol{L})$

\begin{tabular}{|l|l|l|l|l|}
\hline Tipo de falta & $\begin{array}{l}\text { Distância } \\
\text { calculada }(\mathrm{km})\end{array}$ & Erro absoluto $(\mathrm{m})$ & $\begin{array}{l}\text { Erro } \\
(\%)\end{array}$ & $\begin{array}{l}\text { relativo } \\
(\Omega)\end{array}$ \\
\hline $\mathrm{AN}$ & 79,96 & 40 & $-0,029$ & 5,05 \\
\hline $\mathrm{BN}$ & 80,14 & 140 & 0,100 & 4,93 \\
\hline $\mathrm{CN}$ & 80,03 & 30 & 0,021 & 4,98 \\
\hline $\mathrm{ABN}$ & 79,98 & 20 & $-0,014$ & 4,99 \\
\hline $\mathrm{BCN}$ & 80,02 & 20 & 0,014 & 4,97 \\
\hline $\mathrm{CAN}$ & 80,08 & 80 & 0,057 & 4,99 \\
\hline $\mathrm{AB}$ & 79,96 & 40 & $-0,029$ & 4,98 \\
\hline BC & 80,03 & 30 & 0,021 & 4,97 \\
\hline $\mathrm{CA}$ & 79,99 & 10 & $-0,007$ & 5,05 \\
\hline ABC & 80,06 & 60 & 0,043 & 5,00 \\
\hline
\end{tabular}

derivações em linhas pré-existentes, é necessário que sejam disponibilizadas as medições de tensão e corrente por parte das concessionárias ou das empresas de geração.

\section{REFERÊNCIAS BIBLIOGRÁFICAS}

ATP: Alternative Transient Program Rule Book, Leuven EMTP Center, Leuven, K.U., 1987.

BRAHMA, S. M.; GIRGIS A. A. Fault location on a transmission line using synchronized voltage measurements. IEEE Transactions on Power Delivery, v. 19, n. 4, p. 1619 - 1622, Oct. 2004.

FAYBISOVICH, V.; KHOROSHEV, M. I. Frequency domain double-ended method of fault location for transmission lines. In: IEEE/PES. Transmission and Distribution Conference and Exposition, 2008. T\&D. Chicago, IL, 2008. p. 1-6.

FULCZYK, M. et al. Two-end unsynchronized fault location algorithm for double-circuit series compensated lines. In: IEEE. Power and Energy
Tabela 8: Resultados para a variação da resistência de falta para falta no trecho 2 a $50 \mathrm{~km}$ do terminal local $(\boldsymbol{L})$

\begin{tabular}{|l|l|l|l|l|l|}
\hline $\begin{array}{l}\text { Tipo de } \\
\text { falta }\end{array}$ & $\begin{array}{l}\text { Resistência de } \\
\text { falta }(\Omega)\end{array}$ & $\begin{array}{l}\text { Distância } \\
\text { calculada }(\mathrm{km})\end{array}$ & $\begin{array}{l}\text { Erro } \\
\text { absoluto } \\
(\mathrm{m})\end{array}$ & $\begin{array}{l}\text { Erro } \\
\text { relativo } \\
(\%)\end{array}$ & $\begin{array}{l}\text { Resistência } \\
\text { calculada }(\Omega)\end{array}$ \\
\hline \multirow{5}{*}{ Fase-te } & 1 & 50,03 & 30 & 0,030 & 0,97 \\
\cline { 2 - 6 } & 10 & 50,05 & 50 & 0,050 & 9,99 \\
\cline { 2 - 6 } & $\mathbf{r r d} 00$ & 50,15 & 150 & 0,150 & 100,00 \\
\cline { 2 - 6 } & 300 & 50,08 & 80 & 0,080 & 300,00 \\
\hline \multirow{5}{*}{ Trifásica } & 500 & 50,06 & 60 & 0,060 & 499,81 \\
\cline { 2 - 6 } & 1 & 50,01 & 10 & 0,010 & 0,99 \\
\cline { 2 - 6 } & 10 & 50,02 & 20 & 0,020 & 10,00 \\
\cline { 2 - 6 } & 100 & 50,02 & 20 & 0,020 & 99,99 \\
\cline { 2 - 6 } & 300 & 50,02 & 20 & 0,020 & 299,98 \\
\cline { 2 - 6 } & 500 & 50,00 & 0 & 0 & 499,98 \\
\hline
\end{tabular}

Tabela 9: Resultados para a variação da resistência de falta para falta no trecho 4 a $130 \mathrm{~km}$ do terminal local $(\boldsymbol{L})$

\begin{tabular}{|c|c|c|c|c|c|}
\hline $\begin{array}{l}\text { Tipo de } \\
\text { falta }\end{array}$ & $\begin{array}{l}\text { Resistência de } \\
\text { falta }(\Omega)\end{array}$ & $\begin{array}{l}\text { Distância } \\
\text { calculada (km) }\end{array}$ & $\begin{array}{l}\begin{array}{l}\text { Erro } \\
\text { absoluto } \\
\text { (m) }\end{array} \\
\end{array}$ & $\begin{array}{l}\text { Erro } \\
\text { relativo } \\
(\%)\end{array}$ & $\begin{array}{l}\text { Resistência } \\
\text { calculada }(\Omega)\end{array}$ \\
\hline \multirow{5}{*}{ Fase-te } & 1 & 129,96 & 40 & $-0,029$ & 1,05 \\
\hline & 10 & 129,94 & 60 & $-0,043$ & 10,06 \\
\hline & rd00 & 129,93 & 70 & $-0,050$ & 99,90 \\
\hline & 300 & 129,96 & 40 & $-0,029$ & 299,97 \\
\hline & 500 & 129,96 & 40 & $-0,029$ & 499,99 \\
\hline \multirow{5}{*}{ Trifásica } & 1 & 129,93 & 70 & $-0,050$ & 1,01 \\
\hline & 10 & 129,97 & 30 & $-0,021$ & 10,01 \\
\hline & 100 & 130,05 & 50 & $-0,036$ & 99,94 \\
\hline & 300 & 130,16 & 160 & 0,114 & 299,87 \\
\hline & 500 & 129,62 & 380 & $-0,271$ & 500,27 \\
\hline
\end{tabular}

Society General Meeting - Conversion and Delivery of Electrical Energy in the 21st Century. Pittsburgh, PA, 2008. p. 1-9.

GOPALAKRISHNAN, A.; KEZUNOVIC, M.; MCKENNA, S. M.; HAMAI, D. M. Fault location using the distributed parameter transmission line model. IEEE Transactions on Power Delivery, v. 15, $\mathrm{n}$. 4, p. 1169-1174, Oct. 2000.

JEYASURYA, B.; RAHMAN, M.A. Simulation of Transmission Line Fault Locators in a Personal Computer. IEEE Transactions on Industry Applications, v. 27, n. 2, p. 299-302, March-April 1991.

JOHNS, A.T.; MOORE, P.J.; WHITTARD, R. New Technique for the Accurate Location of Earth Faults on Transmission Systems. IEE Proceedings-Generation, Transmission and Distribution, v. 142, n. 2, p. 119-27, March 1995.

LIAO, Y. Fault location for single-circuit line based on bus-impedance matrix utilizing voltage measurements. 
Tabela 10: Resultados para a variação da resistência de falta para falta no trecho 6 a $80 \mathrm{~km}$ do terminal local $(\boldsymbol{L})$

\begin{tabular}{|l|l|l|l|l|l|}
\hline $\begin{array}{l}\text { Tipo de } \\
\text { falta }\end{array}$ & $\begin{array}{l}\text { Resistência de } \\
\text { falta }(\Omega)\end{array}$ & $\begin{array}{l}\text { Distância } \\
\text { calculada }(\mathrm{km})\end{array}$ & $\begin{array}{l}\text { Erro } \\
\text { absoluto } \\
(\mathrm{m})\end{array}$ & $\begin{array}{l}\text { Erro } \\
\text { relativo } \\
(\%)\end{array}$ & $\begin{array}{l}\text { Resistência } \\
\text { calculada }(\Omega)\end{array}$ \\
\hline \multirow{5}{*}{ Fase-terra } & 1 & 79,97 & 30 & $-0,021$ & 1,05 \\
\cline { 2 - 6 } & 10 & 79,95 & 50 & $-0,036$ & 10,06 \\
\cline { 2 - 6 } & 100 & 79,85 & 150 & $-0,107$ & 99,88 \\
\cline { 2 - 6 } & 300 & 80,03 & 30 & 0,021 & 299,62 \\
\cline { 2 - 6 } & 500 & 79,91 & 90 & $-0,064$ & 499,61 \\
\hline \multirow{5}{*}{ Trif́́sica } & 1 & 79,97 & 30 & $-0,021$ & 0,99 \\
\cline { 2 - 6 } & 10 & 79,99 & 10 & $-0,007$ & 10,00 \\
\cline { 2 - 6 } & 100 & 79,97 & 30 & $-0,021$ & 99,97 \\
\cline { 2 - 6 } & 300 & 80,03 & 30 & 0,021 & 299,87 \\
\cline { 2 - 6 } & 500 & 80,03 & 30 & 0,021 & 499,84 \\
\hline
\end{tabular}

IEEE Transactions on Power Delivery, v. 23, n. 2, p. 609-617, April 2008.

LIU, C.; LIEN, K.; CHEN, C.; JIANG, J. A universal fault location technique for $n$-terminal $(n \geq 3)$ transmission lines. IEEE Transactions on Power Delivery, v. 23, n. 3, p. 1366 - 1373, July 2008.

MANASSERO, G. Sistema para localização de faltas em linhas de transmissão com subestações conectadas em derivação. Tese (doutorado), São Paulo, EPUSP, 2006.

MATLAB, High-Performance Numeric Computation and Visualization Software: Reference Guide. Natick, MA: MathWorks, 1992.

NOVOSEL, D.; et al. Algorithms for Locating Faults on Series Compensated Lines Using Neural Network and Deterministic Methods. IEEE Transactions on Power Delivery, v. 11, n. 4, p. 1728-36, Oct. 1996.

TAKAGI, T. et al. Development of a New Type Fault Locator Using The One-Terminal Voltage and Current Data. IEEE Transactions on Power Apparatus and Systems, v. 101, n 8, p. 2892-2898, Aug. 1981.

WANG, B.; DONG X.; BO, Z.; KLIMEK, A. Impedance phase faults location algorithm for DRV transmission lines. In: IEEE/PES. Transmission and Distribution Conference and Exposition: Latin America. Bogotá, Colombia, 2008. p. 1-4. 